\title{
Análisis de las Propiedades Psicometricas del Inventario de Estrategias de Aprendizaje y Estudio (LASSI) en Estudiantes Universitarios
}

\author{
Psychometric Analysis of the Learning and Study Strategies Inventory in College \\ Students
}

\author{
Agustín Freiberg Hoffmann ${ }^{1}$, Ruben Ledesma ${ }^{2}$ y María Mercedes Fernández Liporace ${ }^{3}$
}

\begin{abstract}
Resumen
El trabajo analiza las características psicométricas de la versión local del Inventario de Estrategias de Aprendizaje y Estudio -LASSI- (Fernández Liporace, Scheinsohn, \& Uriel, 2010; Stover, Uriel, \& Fernández Liporace, 2012; Uriel, Stover, Scheinsohn, Contini de González, \& Fernández Liporace, 2009; Weinstein, Schulte, \& Palmer, 1987) para estudiantes universitarios de Buenos Aires. Se redactaron nuevos ítems para sumar a las dimensiones existentes, a fin de ampliar y equiparar la cantidad de reactivos. Además se adicionaron dos dimensiones para evaluar Aprendizaje Colaborativo y Competencias para el Manejo de la Información 2.0. Tras analizar evidencias de validez-contenido, facies, constructo - y confiabilidad consistencia interna, test retest-, se arribó a una estructura factorial que permite evaluar cinco dimensiones Aprendizaje Colaborativo, Motivación, Habilidades para Jerarquizar la Información, Recursos para el Aprendizaje, Competencias para el Manejo de la Información 2.0- mediante 33 elementos. Se discuten los resultados a la luz de cuestiones teóricas.
\end{abstract}

Palabras clave: LASSI, estrategias de aprendizaje, estudiantes universitarios, validez de constructo

\begin{abstract}
The study aims at the analysis of the psychometric features of the local version of the Learning and Study Strategies Inventory -LASSI- (Fernández Liporace, Scheinsohn, \& Uriel, 2010; Stover, Uriel, \& Fernández Liporace, 2012; Uriel, Stover, Scheinsohn, Contini de González, \& Fernández Liporace, 2009; Weinstein, Schulte, \& Palmer, 1987) for college students from Buenos Aires. New items were developed in order to increase the number of propositions in each dimension. Besides, two new dimensions were added to asses Cooperative Learning and Skills to Manage 2.0 Information. After analyzing validity evidences-content, face, construct - and reliability - internal consistency, test retest procedure-, a 5-factor solution was isolated, composed of 33 items. The five dimensions were Cooperative Learning, Motivation, Skills to Distinguish and Organize Information, Learning Resources, and Skills to Manage 2.0 Information. Results are discussed considering theoretical implications.
\end{abstract}

Keywords: LASSI, leaning strategies, college students, construct validity

Agradecimientos: La presente investigación ha sido desarrollada en el marco de una beca postdoctoral otorgada por el Consejo Nacional de Investigaciones Científicas y Técnicas (CONICET).

${ }^{1}$ Doctor en Psicología. Facultad de Psicología, Universidad de Buenos Aires. Consejo Nacional de Investigaciones Científicas y Técnicas (CONICET) (Becario Posdoctoral). Vallejos $23794^{\circ}$ Piso (1419), Argentina. Tel.: 01120713998/1563577196. Correo: afreiberg@psi.uba.ar/agustinfreiberg@gmail.com

2 Doctor en Psicología. Facultad de Psicología, Universidad Nacional de Mar del Plata. Consejo Nacional de Investigaciones Científicas y Técnicas (CONICET) (Investigador Independiente). Rio Negro 3922 (7600). Mar del Plata, Argentina. Tel.: 0223 4716015. Correo: rdledesma@gmail.com

${ }^{3}$ Doctora en Psicología. Facultad de Psicología, Universidad de Buenos Aires. Consejo Nacional de Investigaciones Científicas y Técnicas (CONICET) (Investigadora Independiente). Gral. Juan Lavalle 2353 (1052), Argentina. Tel.: 011 4952-5490. Correo: mliporac@psi.uba.ar 


\section{Introducción}

Toda situación de enseñanza-aprendizaje requiere de parte de los estudiantes, la puesta en marcha de múltiples procesos cognitivos memoria, razonamiento, resolución de problemas, entre otros- que, a su vez, guardan una estrecha relación con las estrategias de aprendizaje y estudio (Peñalosa Castro, Landa Durán, \& Vega Valero, 2006).

Las estrategias de aprendizaje y estudio designan las conductas y pensamientos que las personas emplean para la resolución de tareas específicas a la hora de aprender. Se definen como actividades que se realizan durante la situación de aprendizaje con el propósito de influir sobre los procesos cognoscitivos y afectivos facilitando así la asimilación de conocimientos (Weinstein \& Mayer, 1986). Todas estas actividades se caracterizan por ser intencionales y conscientes, por lo que la selección que se haga de ellas en el momento de lidiar con determinada clase de información depende enteramente del alumno (Beltrán, 2003). Esto ofrece la ventaja de permitir al sujeto trazar un plan de acción de modo tal que, a partir de la información que se desea incorporar, sea posible seleccionar aquellas conductas que faciliten la tarea.

Resulta interesante destacar que las estrategias presentan gran variabilidad interindividual. Así, quienes son capaces de incorporar una mayor variedad de ellas cuentan con un abanico más amplio para seleccionar las que mejor se ajusten a cada situación particular. Contrariamente, es más probable que educandos que emplean una acotada variedad de estrategias adopten acciones poco apropiadas, dificultando la asimilación de información (Cardozo, 2012). Por todo esto conocer las estrategias de aprendizaje del estudiantado se vuelve un punto de interés para el ámbito educativo, en virtud de que ello contribuirá a la identificación de aquellos alumnos con estrategias inapropiadas y/o insuficientes para aprender de manera eficaz (Dunlosky, Rawson, Marsh, Nathan, \& Willingham, 2013).

\section{Estrategias de Aprendizaje en Estudiantes Universitarios}

El interés por conocer el modo en que los estudiantes planifican y administran sus recursos cognitivos ha impulsado el estudio de las estrategias de aprendizaje en su carácter de organizadoras del proceso de adquisición de conocimientos (Kesici, Sahin, \& Akturk, 2009; Weinstein \& Meyer, 1991). Si bien han sido analizadas en diferentes niveles educativos, el universitario ha concentrado un mayor número de investigaciones acumuladas hasta el momento. Varias de ellas han verificado que el empleo de estrategias específicas se halla vinculado a la mejora de los procesos de aprendizaje (e.g. Basnama, Fauziya, Nilofar, Rubina, \& Seema, 2012; Broadbent \& Poon, 2015; Kisac \& Budak, 2014). Además se ha informado que el uso de una mayor variedad de distintas estrategias aumenta a medida que el estudiante avanza en su trayecto académico, adaptándose de este modo a los requerimientos de cada disciplina (e.g. Elvira Valdéz \& Pujol, 2013; Coertjens, Van Daal, Donche, De Maeyer, Vanthounout, \& Van Petegem, 2013).

Por otra parte se ha resaltado la relación entre la puesta en juego de determinadas estrategias y ciertas variables psicológicas, tales como rasgos de personalidad, motivación, ansiedad y flow, así como autorregulación afectiva, conductual y cognitiva, entre otras (Ben Eliyahu \& Linnenbrink Garcia, 2015; Doménech Betoret, Gómez Artiga, \& Lloret Segura, 2014; Kesici, Baloglu, \& Deniz, 2011; Lee \& Choi, 2013; Moldasheva \& Mahmood, 2014; Sullivan, Eduljee, \& Pinkham, 2012).

En cuanto a estrategias y rendimiento académico en estudiantes universitarios, los enfoques cualitativo y cuantitativo proponen vías de abordaje diferenciadas. El primero ubica el acento en la identificación y análisis de estrategias específicas (Cazan, 2012; Diseth, 2011; Gargallo, Almerich, Suárez Rodríguez, \& García Félix, 2012; Kálly, 2012; Yip, 2009), en tanto que el segundo se focaliza en el número de estrategias que los alumnos instrumentan (Bahamón Muñetón, Vianchá Pinzón, Alarcón Alarcón, \& Bohorquéz Olaya, 2013; Fernández González, Martínez Conde Beluzán, \& Melipillán Araneda, 2009; Wenbin, 2006; Zimmerman \& Martínez Pons, 1988).

Con el avance de las nuevas tecnologías se ha puesto de relieve la importancia del manejo de ciertas estrategias vinculadas con el tipo de $\mathrm{N}^{\circ} 44 \cdot \mathrm{Vol} .2 \cdot 116-130 \cdot 2017$ 
aprendizaje a distancia que se utilice - e-learning o b-learning. Se habla así, en primer lugar, de estrategias asociadas al manejo de la información web $2.0 \mathrm{y}$, en segundo término, de estrategias relacionadas con el aprendizaje colaborativo (Moreno Herrero, 2012). Con respecto a la información 2.0, se ha hallado que ciertas estrategias - gestión del tiempo, regulación del esfuerzo y pensamiento crítico, entre otras coinciden con un mayor aprovechamiento de la modalidad e-learning y repercuten directamente en el desempeño (e.g. Broadbent \& Poon, 2015; Cerda, López, Osses, \& Saiz, 2015; Goulão, 2014; Tirziu \& Vrabie, 2015). También se afirma que las estrategias e-learning mejoran la calidad de los aprendizajes a la vez que se tornan necesarias para que los estudiantes participen como agentes activos de esos procesos (e.g. Kovacova \& Vackova, 2015; Valentín, Mateos, González Tablas, Pérez, López, \& García, 2013; Van Seters, Ossevoort, Tramper, \& Goedhart, 2012). Por otro lado, se ha observado que las estrategias orientadas hacia el aprendizaje colaborativo posibilitan el desarrollo de habilidades comunicacionales, de resolución de problemas, e interpersonales (e.g. Sharifah, Khairiyah, Haslenda, \& Zainura, 2012; Wu Yuin, Rustam, Chin Yu, \& Zhi Huan, 2012). Tales estrategias se asocian, además, con un aprovechamiento óptimo de los recursos tecnológicos, así como también con un mejor rendimiento académico (e.g. Delgado Hurtado \& Castrillo Lara, 2014).

Lo mencionado hasta aquí sugiere la pertinencia y utilidad del estudio de las estrategias de aprendizaje en estudiantes universitarios, de cara a la mejora de los procesos de enseñanzaaprendizaje, tanto en contextos de investigación cuanto en el ámbito educativo, en atención a la situación académica individual. El entrenamiento en el manejo de estrategias específicas redunda en una mayor incorporación de los conocimientos, que se traduce en lo que se ha dado en llamar el aprendizaje efectivo (Dunlosky et al., 2013; Weinstein, Ridley, Dahl, \& Weber, 1989). Pero para llegar allí, la instancia previa de evaluación resulta insoslayable.

\section{Evaluación de las Estrategias de Aprendizaje}

$\mathrm{Si}$ bien existen diversos instrumentos diseñados para evaluarlas (e.g. Namlu, 2004;
Pintrich, Smith, García, \& Mckeachie, 1991; Román \& Gallego, 1994), uno de los más clásicos y difundidos es el Inventario de Estrategias de Aprendizaje y Estudio - Learning and Study Strategies Inventory o LASSI - (Weinstein, Schulte, \& Palmer, 1987). Consta de 77 afirmaciones autoadministrables que se responden mediante una escala Likert de cinco opciones, que abarca desde nunca hasta siempre, aludiendo a la frecuencia de uso. Los reactivos se distribuyen en 10 dimensiones teorizadas como independientes Actitud, Motivación, Administración del tiempo, Ansiedad, Concentración, Procesamiento de la información, Selección de ideas principales, Ayudas al estudio, Autoevaluación, y Estrategias de prueba. Este inventario se enfoca en los pensamientos, emociones, comportamientos y actitudes que los individuos reconocen como característicos de su quehacer estudiantil y que pueden, alternativamente, beneficiar o perjudicar sus aprendizajes.

Dada la difusión que el LASSI ha logrado en el ámbito académico, múltiples investigaciones han apuntado a adaptarlo a distintas poblaciones estudiantiles - niveles medio, terciario $\mathrm{y}$ universitarios - provenientes de diferentes contextos (e.g. Badenier, 2006; Cano, 2006; Castañeiras, Guzmán, Posada, Ricchini, \& Strucchi, 1999; Flowers, Bridges, \& Moore, 2011; López Martín, Expósito Casas, González, \& Jiménez García, 2012; Stevens \& Tallent Runnels, 2004; Yip, 2013).

Localmente se ha trabajado una versión para los niveles medio y universitario (Fernández Liporace, Scheinsohn, \& Uriel, 2010; Stover, Uriel, \& Fernández Liporace, 2012; Uriel, Stover, Scheinsohn, Contini de González, \& Fernández Liporace, 2009). Esta versión, ajustada lingüísticamente a los modismos locales, reduce las opciones de respuesta de la escala Likert de cinco a cuatro con el fin de evitar la respuesta neutra. Presenta, además, evidencias de validez de constructo que dan cuenta de una estructura hexafactorial diferente de la propuesta por los autores originales integrada en un primer momento por 10 dimensiones (Weinstein et al., 1987), agrupadas posteriormente en 3 -Habilidad, Voluntad, Autorregulación (Weinstein \& Palmer, 2002). Esta falta de coincidencia entre las dimensiones teorizadas y las extraídas del No44 Vol.2 $116-130 \cdot 2017$ 
procedimiento factorial va en el mismo sentido del resto de los estudios desarrollados (e.g. Badenier, 2006; Cano, 2006; Corral de Zurita \& Alcalá, 2002; Olejnik \& Nist, 1992; Olivarez \& Tallent, 1994; Prevatt, Petscher, Proctor, Hurst, \& Adams, 2006; Samuelstuen, 2003; Valle \& Rodríguez, 1998). Las seis dimensiones en las que se distribuyen los 21 reactivos conservados son Organización y Planificación, Habilidades para el Desempeño en Exámenes, Motivación, Recursos para el Aprendizaje, Estrategias de Control y Consolidación, y Habilidades para Jerarquizar la Información. Cabe destacar que esta versión adolece de una baja consistencia interna (<.80) de sus dimensiones, lo que se explicaría por el escaso número de reactivos que logra conservar cada una de ellas (Stover et al., 2012).

Pese a la difusión del LASSI en diversos contextos debe mencionarse que el mismo carece de actualización en cuanto a su contenido, lo cual resulta necesario dado el avance de las nuevas tecnologías. Esto representa otro punto débil de la herramienta, que como tal debe ser abordado a fin de adaptarla a las características del ámbito educativo actual.

A partir, entonces, de esta versión local y en vista de la importancia que el estudio de las estrategias de aprendizaje en estudiantes universitarios reviste, esta investigación se propone: 1) Ampliar el número de reactivos de las dimensiones derivadas de la versión adaptada localmente, 2) Desarrollar dos nuevas escalas para sumar a las seis existentes, a fin de valorar estrategias ligadas al manejo de la información 2.0, y al aprendizaje colaborativo, 3) Analizar evidencias de validez de contenido y aparente del instrumento, 4) Examinar evidencias de validez de constructo, 5) Estudiar, para cada una de las dimensiones, la consistencia interna de las puntuaciones, así como también su estabilidad temporal.

\section{Método}

\section{Diseño de Investigación}

Se llevó a cabo un estudio correlacionalexplicativo de corte transversal (Nieto Martín \& Rodríguez Conde, 2010).

\section{Participantes}

Estudio de validez de contenido y aparente. Tres psicólogos educacionales fueron convocados para oficiar de jueces expertos en la evaluación del contenido de los elementos. Adicionalmente se contó con la participación de 20 estudiantes universitarios de la Facultad de Psicología para la realización del estudio piloto.

Estudio de validez de constructo y consistencia interna. Los estudios factoriales exploratorio y confirmatorio se efectuaron a partir de dos muestras diferentes de estudiantes universitarios de Buenos Aires, de modo de garantizar la independencia de los datos en ambas instancias de análisis.

- Estudio factorial exploratorio: Participaron 337 estudiantes universitarios (42.7\% varones; $57.3 \%$ mujeres) de tres unidades académicas de Buenos Aires (49.6\% Psicología, 29.7\% Ingeniería, 20.8\% Derecho). Las edades variaron entre 17 y 36 años $(\bar{X}=22.69 ; D E=3.93)$.

- Estudio factorial confirmatorio: Se analizó una muestra de 402 estudiantes universitarios (57\% varones; $43 \%$ mujeres) de distintas facultades (35.1\% Psicología, 29.9\% Ingeniería, 29.9\% Exactas y Naturales, 5.2\% Medicina) ubicadas en Buenos Aires. Las edades se movían entre los 18 y $\operatorname{los} 36$ años ( $\bar{X}=22.79 ; D E=3.35$ ).

Estudio de confiabilidad test-retest. Se trabajó con 19 estudiantes de Psicología (20\% varones y $80 \%$ mujeres), entre 21 y 36 años de edad $(\bar{X}$ $=24.85 ; D E=4.17$ ).

\section{Instrumentos}

Se utilizó una encuesta sociodemográfica y académica para recoger datos descriptivos sobre los alumnos -sexo, edad, universidad, facultad y carrera- y recabar información adicional -grupo de convivencia, educación y profesión parental, y tipo de educación media (bachiller o técnico)- que permitiera valorar la validez interna relativa a la recolección de datos. Se excluyeron los casos en los se omitió más de la mitad de los datos sociodemográficos y académicos, tomándose ello como indicador de falta de interés y de cooperación.

Se aplicó el Inventario de Estrategias de Aprendizaje y Estudio (LASSI) (Weinstein et al., 1987) en su versión adaptada a estudiantes medios y universitarios de Buenos Aires (Fernández 
Liporace et al., 2010; Stover et al., 2012; Uriel et al., 2009). A los 21 reactivos de esta versión se adicionaron 64 nuevos elementos, de los cuales 39 se redactaron con el propósito de incrementar y emparejar el número de ítems de las escalas existentes, quedando conformada cada una de ellas por 10 afirmaciones. Los otros 25 ítems fueron diseñados para evaluar las nuevas dimensiones propuestas -15 para estrategias para el manejo de la información 2.0, y 10 para la de aprendizaje colaborativo. Así el inventario resultante quedó constituido por un total de 85 elementos.

\section{Procedimientos}

Estudio de validez de contenido y aparente. Los tres jueces expertos recibieron un instructivo que contenía una breve reseña teórica, la descripción de cada estrategia y las consignas para completar la tarea. Además se les entregó un cuadernillo en el que se encontraban ordenados aleatoriamente los 85 elementos - 21 correspondientes a la versión abreviada y 64 redactados para ampliar el número de reactivos de cada dimensión y para evaluar las dos nuevas. Adicionalmente el cuadernillo presentaba dos escalas en las que los expertos debían indicar, según su criterio, la dimensión a la que pertenecía cada elemento y la calidad de cada uno con respecto a la dimensión según una escala de uno a cinco, donde uno representaba nada adecuado y cinco, extremadamente adecuado (Moreno Bayardo, 2000). Los reactivos que se conservaron luego de esta instancia fueron empleados para conformar la versión que fue sometida luego al estudio piloto a fin de valorar la comprensión de los elementos y de la consigna.

\section{Estudio de validez de constructo y consistencia} interna. Un psicólogo habilitado y debidamente entrenado realizó la administración colectiva de los instrumentos durante el horario de clases. Los estudiantes que participaron respondiendo a los instrumentos lo hicieron de modo voluntario. Todos ellos firmaron un consentimiento informado y se les garantizó el anonimato de los datos en todo momento. El estudio contó con los respectivos avales institucionales.
Estudio de Confiabilidad Test-retest. Se trabajó con dos administraciones separadas por un intervalo de 30 días.

\section{Análisis de Datos}

Estudio de validez de contenido. El acuerdo entre jueces fue valorado a través del coeficiente $\mathrm{V}$ de Aiken. Su cálculo se realizó usando un programa diseñado en Visual Basic (Merino Soto \& Livia Segovia, 2009).

Estudio de validez de constructo y consistencia interna. Dada la naturaleza politómica de las respuestas previstas se decidió emplear aplicativos estadísticos acordes al tratamiento de ese tipo de datos, que posibilitaran la ejecución de los análisis a partir de matrices de correlaciones policóricas (Manzano Patiño \& Zamora Muñoz, 2009; Richaud de Minzi, 2005). Para el estudio exploratorio y de consistencia interna se utilizó el programa FACTOR 10.1. Para el análisis factorial confirmatorio, el software EQS 6.2.

Estudio de confiabilidad test-retest. La estabilidad temporal de las puntuaciones fue evaluada mediante el paquete estadístico SPSS 21, correlacionando las puntuaciones de la primera y de la segunda administración de cada dimensión del LASSI.

\section{Resultados}

Estudio de validez de contenido y aparente. Se emplearon dos criterios rectores para el análisis del contenido de los reactivos, determinando su conservación o rechazo. El primero rezaba que al menos dos de los tres jueces convocados hubieran clasificado el ítem analizado en una misma dimensión. El segundo, que el coeficiente $\mathrm{V}$ de Aiken del elemento que hubiese cumplido con la instancia anterior superara el valor de .60. Debe mencionarse que los valores de este índice, que se emplea para cuantificar la validez de contenido, fluctúan entre cero y uno, indicando cero la falta absoluta de acuerdo y uno, un perfecto acuerdo con respecto al contenido del elemento (Merino Soto \& Livia Segovia, 2009).

Como resultado del juicio experto se conservaron 62 de los 85 reactivos sometidos a evaluación, que se repartieron en las dimensiones del siguiente modo: 5 en Organización y 
Planificación, 6 en Habilidades para el Desempeño en Exámenes, 10 para Motivación, 3 para Recursos para el Aprendizaje, 10 para Estrategias de Control y Consolidación, 7 para Habilidades para Jerarquizar la Información, 9 para Competencias para el Manejo de la Información 2.0, y 12 para Aprendizaje Colaborativo.

Los ítems derivados del procedimiento anterior fueron posteriormente ordenados aleatoriamente, conformado una nueva versión del instrumento que fue sometida a un examen de validez aparente. Se administró a un grupo de 20 estudiantes universitarios, tomándose como criterio para efectuar modificaciones que al menos cuatro alumnos hubieran coincidido en alguna sugerencia. Si bien se obtuvieron algunas recomendaciones, no se registraron propuestas consensuadas por lo que no se practicaron modificaciones en esta instancia.

\section{Estudio de validez de constructo}

- Estudio factorial exploratorio y análisis de consistencia interna. Se aplicó un análisis de componentes principales ensayándose diversas soluciones, esperando reproducir empíricamente la estructura obtenida para la versión local hexafactorial más las dos dimensiones añadidas. Tras extraer varias estructuras factoriales posibles, se arribó a una de cinco componentes, que presentaba el mejor ajuste y el mejor equilibrio entre representatividad y parsimonia teórica, integrada por las dimensiones Motivación, Recursos para el Aprendizaje, Habilidades para Jerarquizar la Información, Competencias para el Manejo de la Información 2.0, y Aprendizaje Colaborativo. Quedaron excluidas las dimensiones Organización y Planificación, Habilidades para el Desempeño de Exámenes, y Estrategias de Control y Consolidación. El análisis se realizó con rotación Várimax y normalización Kaiser, observándose un razonable ajuste y balance entre el número de sujetos y de ítems $(\mathrm{KMO}=.772$; Test de Esfericidad de Bartlett: $\mathrm{X}^{2}=3542.8 \mathrm{gl} ; 528 \mathrm{sig}<.01$ ). Fueron eliminados todos los reactivos cuya carga en un factor no superara el valor de .40 (Valderrey Sanz, 2010), así como también aquellos que presentaran saturaciones simultáneas por encima de ese valor (Vallejo Seco, 1992).

Revista Iberoamericana de Diagnóstico y Evaluación - e Avaliação Psicológica. RIDEP · N44 · Vol.2 $\cdot 116-130 \cdot 2017$
Los cinco componentes aislados dieron cuenta del $49.8 \%$ de la varianza total, explicando el Factor 1 el $15.1 \%$, el Factor 2 el $11.7 \%$, el Factor 3 el $9.1 \%$, el Factor 4 el $7.9 \%$ y el 5 el $6 \%$, conservándose un total de 33 elementos de los 62 inicialmente sometidos a análisis (Tabla 1).

Tabla 1. Estructura factorial del LASSI, adaptación local y actualizada

\begin{tabular}{|c|c|c|c|c|c|}
\hline \multirow[b]{2}{*}{ Ítems } & \multicolumn{5}{|c|}{ Componentes } \\
\hline & $\begin{array}{c}\text { Factor } \\
1 \\
\text { (AC) }\end{array}$ & $\begin{array}{c}\text { Factor } \\
2 \\
(\mathrm{M}) \\
\end{array}$ & $\begin{array}{c}\text { Factor } \\
3 \\
(\mathrm{HJI}) \\
\end{array}$ & $\begin{array}{c}\text { Factor } \\
4 \\
\text { (RA) }\end{array}$ & $\begin{array}{c}\text { Factor } \\
5 \\
(\mathrm{CMI})\end{array}$ \\
\hline LASSI19 & .748 & & & & \\
\hline LASSI28 & .547 & & & & \\
\hline LASSI29 & .571 & & & & \\
\hline LASSI30 & .782 & & & & \\
\hline LASSI33 & .772 & & & & \\
\hline LASSI34 & .672 & & & & \\
\hline LASSI41 & .593 & & & & \\
\hline LASSI42 & .773 & & & & \\
\hline LASSI60 & .640 & & & & \\
\hline LASSI62 & .751 & & & & \\
\hline LASSI05 & & .783 & & & \\
\hline LASSI09 & & .654 & & & \\
\hline LASSI17 & & .716 & & & \\
\hline LASSI 21 & & .623 & & & \\
\hline LASSI26 & & .529 & & & \\
\hline LASSI27 & & .506 & & & \\
\hline LASSI37 & & .522 & & & \\
\hline LASSI56 & & .789 & & & \\
\hline LASSI02 & & & .644 & & \\
\hline LASSI18 & & & .689 & & \\
\hline LASSI45 & & & .653 & & \\
\hline LASSI46 & & & .707 & & \\
\hline LASSI47 & & & .698 & & \\
\hline LASSI54 & & & .628 & & \\
\hline LASSI08 & & & & .564 & \\
\hline LASSI24 & & & & .825 & \\
\hline LASSI35 & & & & .833 & \\
\hline LASSI43 & & & & .485 & \\
\hline LASSI58 & & & & .647 & \\
\hline LASSI03 & & & & & .624 \\
\hline LASSI20 & & & & & .752 \\
\hline LASSI39 & & & & & .498 \\
\hline LASSI51 & & & & & .662 \\
\hline
\end{tabular}


Tabla 2. LASSI. Ítems que saturaron en una dimensión diferente respecto de la versión local de Stover, Uriel y Fernández Liporace (2012)

\begin{tabular}{|c|c|c|}
\hline Ítems & $\begin{array}{c}\text { Dimensión } \\
\text { Original } \\
\end{array}$ & $\begin{array}{l}\text { Dimensión } \\
\text { Saturación }\end{array}$ \\
\hline $\begin{array}{l}\text { 08. Armo resúmenes que articulen el } \\
\text { contenido de varios textos. }\end{array}$ & ECC & RA \\
\hline $\begin{array}{l}\text { 26. Articulo la información que me dan en } \\
\text { clase con la que leo de los textos. }\end{array}$ & ECC & $\mathrm{M}$ \\
\hline $\begin{array}{l}\text { 37. Si tengo alguna duda en relación a un } \\
\text { tema vuelvo a consultar los apuntes y/o } \\
\text { textos. }\end{array}$ & ECC & M \\
\hline $\begin{array}{l}\text { 43. Practico ejercicios similares a los que se } \\
\text { van a tomar en los exámenes. }\end{array}$ & ECC & RA \\
\hline $\begin{array}{l}\text { 51. Suelo quedarme después de clases } \\
\text { conversando con mis compañeros sobre los } \\
\text { temas que se vieron ese día. }\end{array}$ & $\mathrm{AC}$ & CMI \\
\hline
\end{tabular}

Del examen de los reactivos que componen cada factor se advierte que 5 del total analizado saturaron en una dimensión diferente de aquella en la que fueran incluidos en el trabajo previo de Stover, Uriel y Fernández Liporace (2012) (Tabla 2). Sin embargo, la lectura de su contenido indicó una coherencia entre ese aspecto y la carga factorial obtenida en el presente estudio.

Teniendo en cuenta el nivel de medición previsto para las respuestas a los ítems se decidió analizar la consistencia interna de cada dimensión calculando sendos coeficientes alfa ordinales a partir de correlaciones policóricas, a fin de evitar posibles infraestimaciones (Elosua Oliden \& Zumbo, 2008; Gadermann, Guhn, \& Zumbo, 2012). Se obtuvo un índice de .84 para el Factor 1 (AC), .78 para el Factor 2 (M), .73 para el Factor 3 (HJI), .72 para el Factor 4 (RA) y .54 para el Factor 5 (CMI).

- Estudio factorial confirmatorio. Se calculó un análisis factorial confirmatorio con el fin de verificar el ajuste de las relaciones entre variables latentes y observadas en el modelo pentafactorial obtenido en el análisis exploratorio (Figura 1). Debido a la naturaleza ordinal de las variables observadas se decidió aplicar una metodología robusta, partiendo de una matriz de correlación policórica sobre la que se calculó la respectiva matriz de covarianzas (Christoffersson, 1975; Juras \& Pasaric, 2006; Richaud de Minzi, 2005). Se usó el método de estimación de mínimos cuadrados generalizados asintóticos (AGLS), apropiado para el tratamiento de variables categóricas en muestras amplias ( $\mathrm{N}>200)$ (Bentler, 2006; Byrne, 2008; Schermelleh-Engel, Moosbrugger, \& Müller, 2003). La evaluación del ajuste del modelo se efectuó a través de la lectura de diversos índices de bondad de ajuste

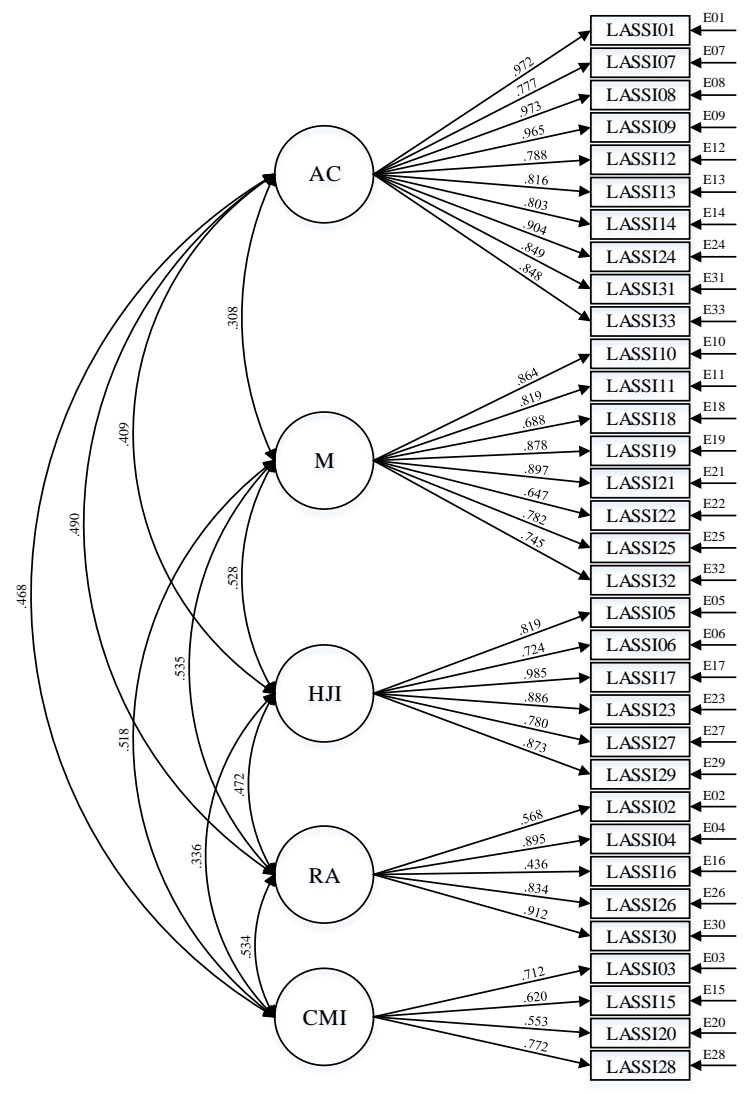

Figura 1. LASSI. Modelo Pentafactorial 
(e.g. Holgado Tello, Chacón Moscoso, Barbero García, \& Vila Abad, 2010; Jöreskog \& Sörbom, 1999; Kline, 2005), siendo tales NFI (Normed Fit Index), NNFI (Non-Normed Fit Index), CFI (Comparative Fit Index), IFI (Incremental Fit Index), RMSEA (Root Mean Square Error of Approximation). Los resultados mostraron valores superiores a .90 para NFI, NNFI, CFI e IFI, guarismo consensuado como punto límite entre un buen ajuste y uno mediocre (Brown, 2015; Byrne, 1998; Harrington, 2009). Con respecto a RMSEA también se obtuvo un valor adecuado, ubicado entre 0 y .08 (Lévy Mangin \& González, 2006) (Tabla 3).

Tabla 3. Índices de ajuste del modelo pentafactorial del inventario LASSI

\begin{tabular}{cccccc}
\hline \multirow{3}{*}{ Modelo } & \multicolumn{5}{c}{ Índices de Ajuste } \\
\cline { 2 - 6 } & NFI & NNFI & CFI & IFI & $\begin{array}{c}\text { RMSEA } \\
{[\text { IC] }}\end{array}$ \\
\hline Pentafactorial & .928 & .960 & .963 & .963 & $\begin{array}{c}.049 \\
{[.044-} \\
.054]\end{array}$ \\
\hline
\end{tabular}

Pasando al análisis de los parámetros estimados, no se registraron ecuaciones infractoras (casos Heywood y parámetros estandarizados superiores a 1), lo que indica una adecuada validez externa del modelo (Flora \& Curran, 2004). Además los parámetros estandarizados de las variables observadas han sido todos significativos $(p<.05)$, alcanzando en 26 de los 33 reactivos valores superiores a .70 , corte que se traduce en coeficientes de determinación aceptables $\left(\mathrm{R}^{2}>.50\right)$ (Kline, 2005) (Tabla 4). Cabe aclarar que el coeficiente de determinación expresa la relación que guardan las variables observadas con las latentes, permitiendo ponderar el aporte de cada reactivo a la explicación de la varianza.

Posteriormente se examinaron las covarianzas a fin de analizar evidencias de validez convergente y discriminante entre las dimensiones integrantes del modelo. De este modo se consideraron aquellos valores superiores a .85 como indicadores de evidencias de validez convergente entre factores, sugiriendo la posibilidad de fundir ambas en una, y valores por debajo de .50 como representativos de evidencias de validez discriminantes, señalando la alternativa de agregar variables latentes al modelo (Rial Boubeta, Varela Mallou, Abalo Piñeiro, \& Lévy Mangin, 2006). A partir de la figura 1 es posible observar que de las 10 covarianzas que integraban el modelo, 4 se encontraban entre ambos cortes de referencia siendo tales M-HJI (.528), M-RA (.535), M-CMI (.518) y RA-CMI (.534). Dos covarianzas se ubicaron en valores muy cercanos a .50, las cuales fueron AC-RA (.490) y AC-CMI (.468). Finalmente cuatro covarianzas adoptaron valores claramente inferiores a .50 , dando cuenta de evidencias de validez discriminante. Estas fueron AC-HJI (.409), AC-M (.308), HJI-CMI (.336) y HJI-RA (.336). No se observaron coeficientes superiores a .85 que indicaran la pertinencia de unificar dimensiones.

Finalmente y de acuerdo al nivel de medición ordinal de las variables observables se analizó nuevamente la consistencia interna de cada dimensión calculando para todas ellas los respectivos coeficientes alfa ordinales (Elosua Oliden \& Zumbo, 2008; Gadermann et al., 2012).

Tabla 4. Coeficientes de determinación para cada reactivo del inventario LASSI, versión local

\begin{tabular}{cccccccc}
\hline Ítem & $\mathrm{R}^{2}$ & Ítem & $\mathrm{R}^{2}$ & Ítem & $\mathrm{R}^{2}$ & Ítem & $\mathrm{R}^{2}$ \\
\hline LASSI01 & .945 & LASSI11 & .671 & LASSI21 & .805 & LASSI31 & .721 \\
LASSI02 & .323 & LASSI12 & .306 & LASSI22 & .419 & LASSI32 & .555 \\
LASSI03 & .508 & LASSI13 & .665 & LASSI23 & .784 & LASSI33 & .720 \\
LASSI04 & .802 & LASSI14 & .644 & LASSI24 & .817 & & \\
LASSI05 & .671 & LASSI15 & .384 & LASSI25 & .611 & & \\
LASSI06 & .508 & LASSI16 & .190 & LASSI26 & .695 & & \\
LASSI07 & .603 & LASSI17 & .969 & LASSI27 & .609 & & \\
LASSI08 & .946 & LASSI18 & .474 & LASSI28 & .596 & & \\
LASSI09 & .931 & LASSI19 & .770 & LASSI29 & .761 & & \\
LASSI10 & .746 & LASSI20 & .306 & LASSI30 & .832 & &
\end{tabular}


Se obtuvo un índice de .97 para Aprendizaje Colaborativo, .93 para Motivación, .94 para Habilidades para Jerarquizar la Información, .85 Recursos para el Aprendizaje y .76 para Competencias para el Manejo de la Información 2.0.

- Estudio de confiabilidad test-retest. La versión abreviada resultante de los análisis de validez de constructo fue posteriormente examinada en cuanto a la estabilidad a 30 días de cada una de las puntuaciones consideradas. El estudio correlacional entre los valores de la primera y segunda administración arrojó resultados aceptables -aunque no óptimos en todas las dimensiones- y significativos $(p<.01)$ de .79 para el Factor 1 (Aprendizaje Colaborativo), .77 para el Factor 2 (Motivación), 89 para el Factor 3 (Habilidades para Jerarquizar la Información), .73 para el Factor 4 (Recursos para el Aprendizaje), y .75 para el Factor 5 (Competencias para el Manejo de la Información 2.0).

\section{Discusión}

Con el propósito de mejorar las propiedades técnicas de la versión local para población universitaria del inventario LASSI el presente trabajo se propuso ampliar el número de reactivos en las dimensiones del instrumento y diseñar dos dimensiones que evaluaran estrategias vinculadas al aprendizaje colaborativo y a las competencias para el manejo de la información 2.0., analizando indicadores psicométricos a fin de lograr una versión mejorada y actualizada a los parámetros educativos actuales.

El instrumento resultante de los análisis desarrollados permite evaluar cinco de las ocho dimensiones inicialmente puestas a prueba, las cuales, a la vez, han demostrado poseer una desigual relevancia en cuanto a la explicación de las estrategias de aprendizaje. En este sentido el factor más importante es el Aprendizaje Colaborativo que explica un $15.1 \%$, seguido por Motivación con el $11.7 \%$, luego Habilidades para Jerarquizar la Información aporta un 9.1\%, Recursos para el Aprendizaje un 7.9\% y Competencias para el Manejo de la Información 2.0., describe el 6\%. Si bien estos porcentuales no muestran diferencias dramáticas entre sí, podría pensarse que el aprendizaje en grupos aparece como un factor fundamental a la hora de analizar las estrategias, seguida de cerca por vectores motivacionales. La capacidad explicativa más baja se advierte en la destreza en el manejo de medios tecnológicos para la gestión de la información, cuestión que probablemente esté vinculada a lo incipiente del desarrollo de estos recursos en el sistema educativo local, donde aún predomina la modalidad presencial con escasos agregados de $b$ learning y más escasos casos aún, de e-learning. Hasta aquí los pares aparecen como factores clave en actividades de aprendizaje y estudio, información que resulta de utilidad para planificaciones didácticas y diseños de programas de estudio.

En cuanto a las dimensiones incluidas en estudios precedentes (Fernández Liporace et al., 2010; Stover et al., 2012; Uriel et al., 2009) Organización y Planificación, Habilidades para el Desempeño de Exámenes, y Estrategias de Control y Consolidación, no obtuvieron aquí correlato empírico, quedando excluidas de la versión derivada del estudio exploratorio. $\mathrm{Su}$ ausencia podría explicarse a partir de la falta de convergencia de los elementos de cada dimensión, dificultad que puede ligarse al contenido de los mismos ya que el punto de corte del coeficiente $\mathrm{V}$ de Aiken adoptado para decidir la conservación de cada ítem fue de .60, pudiéndoselo considerar como medianamente exigente. $\mathrm{Si}$ bien esta decisión permitió conservar un elevado número de ítems luego del procedimiento de juicio experto (62 de los 85 ítems), pareciera que no todos ellos presentaban un contenido claramente definido, lo que posteriormente pudo haberse visto reflejado en el estudio exploratorio.

Por otra parte y como ya se ha mencionado, la mayoría de las investigaciones que analizaron evidencias de validez de constructo del instrumento desde su diseño hasta la fecha no han conseguido reproducir la estructura factorial propuesta por sus autores (Weinstein et al, 1987). En su lugar se ha arribando a múltiples soluciones distintas del modelo originalmente propuesto (e.g. Badenier, 2006; Cano, 2006; Corral de Zurita \& Alcalá, 2002; Olejnik \& Nist, 1992; Olivarez \& Tallent, 1994; Prevatt et al., 2006; Samuelstuen, 2003; Valle \& Rodríguez, 1998). Los resultados aquí reportados se mueven en idéntica dirección. 
Todo esto reafirma las dudas que el modelo subyacente a la escala genera, más allá de su reconocida utilidad práctica y su difusión en ámbitos educativos y de investigación.

En relación con la consistencia interna de cada una de las dimensiones los resultados del estudio exploratorio arrojaron sólo un índice óptimo (>.80) para el factor Aprendizaje Colaborativo, y coeficientes aceptables (entre .70 y .80 ) en los componentes Motivación, Habilidades para Jerarquizar la Información, y Recursos para el Aprendizaje. Finalmente el factor etiquetado como Competencias para el Manejo de la Información 2.0 obtuvo un índice de consistencia interna de .54 que, si bien es bajo, puede ser considerado admisible teniendo en cuenta el escaso número de reactivos que logró conservar (Martínez Arias, 2005). Debido a que las puntuaciones del procedimiento exploratorio se ven fuertemente afectadas por el error de medida, es que se decidió replicar el análisis en la instancia confirmatoria la cual permite estimar la consistencia interna de las dimensiones mediante un procedimiento de modelización que posibilita analizar por separado la varianza explicada de cada ítem con independencia de la varianza de error (Herrero, 2010). Se obtuvieron así coeficientes óptimos para las estrategias de Aprendizaje Colaborativo, Motivación, Habilidades para Jerarquizar la Información y Recursos para el Aprendizaje y un valor cercano al óptimo (.76) en Competencias para el Manejo de la Información 2.0. Se observa así, en términos generales, una adecuada consistencia interna de todas las dimensiones evaluadas.

En el análisis consecuente de los ítems se observó que todos ellos se relacionaron significativamente con las variables latentes para las que fueron diseñados. Además se ha puesto en evidencia que 26 de los 33 reactivos realizan un aporte sustancial a la explicación de las variables latentes $\left(\mathrm{R}^{2}>.50\right)$. Con respecto a los siete ítems que no llegan a aportar el 50\% de su varianza a la explicación de los factores, se ha optado en el presente estudio por no realizar ningún tratamiento sobre los mismos. Esta decisión se basó en que intervenciones tales como eliminar ítems suelen efectuarse con el propósito de mejorar el ajuste del modelo, que no se ha visto perjudicado por la presencia de tales variables Revista Iberoamericana de Diagnóstico y Evaluación - e Avaliação Psicológica. RIDEP · No44 · Vol.2 · 116-130 2017
(Brown, 2015). Además las acciones vinculadas con la reespecificación de modelos (por ejemplo la supresión de ítems) son desaconsejadas ya que convierten al procedimiento confirmatorio en uno exploratorio (Byrne, 2008). Se espera así examinar en futuras investigaciones tanto los índices de modificación, como también la matriz de residuos normalizados, a fin de determinar la eliminación de esas variables, o bien la revisión y mejora del contenido de las mismas (Rial Boubeta et al., 2006).

Finalmente el análisis de las covarianzas permite afirmar que existe una relativa independencia entre las variables latentes. Solamente cuatro de las diez covarianzas que integran el modelo presentaron valores vinculados a evidencias de validez discriminante entre variables. Ellas fueron Aprendizaje ColaborativoHabilidades para Jerarquizar la Información (.409), Aprendizaje Colaborativo-Motivación (.308), Habilidades para Jerarquizar la Información-Competencias para el Manejo de la Información (.336) y Habilidades para Jerarquizar la Información-Recursos para el Aprendizaje (.336). Si bien las variables latentes en análisis evalúan diferentes tipos de estrategias, no necesariamente se espera que haya diferencias marcadas entre ellas ya que a su vez apuntan a valorar un constructo general, que son las estrategias de aprendizaje. Estos valores sugieren la necesidad de incluir variables intermedias, siempre que tales incorporaciones sean coherentes con el modelo (Rial Boubeta et al., 2006). No obstante tales modificaciones hubieran requerido reespecificar el modelo, acción que, como ya se ha mencionado excede los propósitos del presente estudio, además de no ser ello necesario cuando se han obtenido apropiados índices de ajuste.

Con respecto a la estabilidad temporal de las puntuaciones de cada dimensión es posible afirmar que se han encontrado valores apropiados $(\mathrm{r}>.70)$, aunque no óptimos $(\mathrm{r}<.80)$, que estarían indicando que las estrategias no permanecen totalmente estables a lo largo del tiempo sino que varían levemente. Esto resulta lógico ya que, como se ha comentado en el apartado teórico, estudios previos han dado cuenta de variaciones de tal naturaleza en estudiantes universitarios, quienes tienden a adaptar constantemente las estrategias a los contenidos académicos, y según 
su estado de avance en la carrera (Elvira Valdéz \& Pujol, 2013; Coertjens et al., 2013).

En cuanto a las limitaciones del estudio debe señalarse, en primer lugar, el haber empleado un valor de coeficiente $\mathrm{V}$ de Aiken poco exigente. Esa decisión conservadora, tendiente a retener un elevado número de elementos para someterlos posteriormente al estudio exploratorio, tuvo su desventaja en la mayor longitud del inventario pudiendo ello haber influido en la predisposición de los participantes a responderlo. Además, como ya se ha destacado, tal decisión pudo haber afectado los resultados del análisis exploratorio posterior, debido a que la imprecisión de las formulaciones podría estar obrando en detrimento de la adecuada comprensión por parte de los estudiantes.

En relación con el estudio exploratorio puede nombrarse la escasa diversidad de carreras que integraron la muestra, restricción que pudo haber repercutido en los resultados que, a diferencia de los provenientes de análisis factoriales confirmatorios, dependen fundamentalmente de esa variabilidad muestral (Byrne, 2008).

Respecto de otras características muestrales debe aclararse primeramente que el estudio confirmatorio contó con un número de participantes apropiado $(\mathrm{N}>200)$ dada la cantidad y las características de las variables (Brown, 2015). No obstante el tamaño muestral no fue lo suficientemente grande para realizar estudios de validación cruzada para lo cual se requieren tamaños mayores para la división en submuestras, y evitar así errores de convergencia de las matrices de covarianzas. Asimismo es probable que los bajos coeficientes de determinación de los siete reactivos e indicios de evidencias de validez discriminante en tres covarianzas puedan deberse al tamaño muestral (Rial Boubeta et al., 2006). La incorporación de nuevos casos intentará corregir estas vicisitudes en el futuro próximo, a fin de intentar mejores ajustes en los parámetros estimados y de efectuar los estudios de validación cruzada requeridos.

Para finalizar, como resultado de los distintos análisis se obtuvo un inventario de breve administración cuyo empleo posibilitará a los profesionales psicólogos del ámbito educativo evaluar las estrategias de aprendizaje de los alumnos. Se espera que la información generada Revista Iberoamericana de Diagnóstico y Evaluación - e Avaliação Psicológica. RIDEP · No44 · Vol.2 · 116-130 · 2017 permita planificar acciones tendientes a motivar el empleo de estrategias poco presentes en los educandos, de cara a la facilitación de la asimilación de conocimientos. Por otra parte el carácter intencional y consciente de las estrategias vuelve aún más valiosa la información provista por el inventario. El conocimiento que los estudiantes puedan incorporar acerca de aquellas les será de utilidad para conocer los propios recursos cognitivos y afectivos para hacer frente de las diferentes situaciones de aprendizaje.

\section{Referencias}

Badenier, C. (2006). Confiabilidad y validez del Learning and Study Strategies Inventory (LASSI) en una muestra de estudiantes de la región metropolitana. Psyhke, 12(2), 193-206.

Bahamón Muñetón, M. J., Vianchá Pinzón, M. A., Alarcón Alarcón, L. L., \& Bohorquéz Olaya, C. I. (2013). Estilos y estrategias de aprendizaje relacionadas con el logro académico en estudiantes universitarios. Pensamiento Psicológico, 11(1), 115-129.

Basnama, A.K., Fauziya, A., Nilofar, V., Rubina, B., \& Seema, R. (2012). Students' perception of clinical teaching and learning strategies: A pakistani perspective. Nurse Education Today, 32(1), 85-90. doi:10.1016/j.nedt.2011.01.016

Beltrán, J. (2003). El aprendizaje: Nuevas aportaciones. Revista de Educación, 332, 5573.

Ben Eliyahu, A., \& Linnenbrink Garcia, L. (2015). Integrating the regulation of affect, behavior, and cognition into self-regulated learning paradigms among secondary and post-secondary students. Metacognition and Learning, 10(1), 15-42. doi: http://dx.doi.org/ 10.1007/s11409-014-9129-8

Bentler, P.M. (2006). EQS 6 structural equation program manual. Encico, CA: Multivariate Software, Inc.

Broadbent, J., \& Poon, W. L. (2015). Selfregulated learning strategies \& academic achievement in on line higher education learning environments: A systematic review. The Internet and Higher Education, 27, 1-13. doi:10.1016/j.iheduc.2015.04.007 
Brown, T. A. (2015). Confirmatory factor analysis for applied research. New York: Guilford Press.

Byrne, B. (2008). Structural equation modeling with EQS. New Jersey: Psychology Press.

Byrne, B. M. (1998). Structural Equation Modeling with LISREL, PRELIS and SIMPLIS. London: Lawrence Erlbaum Associates, Inc.

Cano, F. (2006). An in-depth analysis of the Learning and Study Strategies Inventory (LASSI). Educational and Psychological Measurement, 66(6), 1023-1038. doi: $10.1177 / 0013164406288167$

Cardozo, A. (2012). Estilos y estrategias de aprendizaje ¿Constructos complementarios o diferentes? Trabajo presentado en el $V$ Congreso Mundial de Estilos de Aprendizaje, Santander, España. Recuperado de http://dialnet.unirioja.es/descarga/articulo/463 6928.pdf

Castañeiras, C., Guzmán, G., Posada, M.C., Ricchini, M., \& Strucchi, E. (1999). Sobre estrategias de aprendizaje y hábitos de estudio. Baremación Marplatense. Revista Iberoamericana de Diagnóstico y Evaluación - e Avaliação Psicológica, 8(2), 37-50.

Cazan, A. M. (2012). Self-regulated learning strategies-predictors of academic adjustment. Procedia-Social and Behavioral Sciences, 33, 104-108. doi: 10.1016/j.sbspro.2012.01.092

Cerda, C., López, O., Osses, S., \& Saiz, J. (2015). Análisis psicométrico de la Escala de Aprendizaje de Autodirigido basada en la teoría de Aprendizaje Autodirigido de Garrison. Revista Iberoamericana de Diagnóstico y Evaluación - e Avaliação Psicológica, 39, 46-56.

Christoffersson, A. (1975). Factor analysis of dichotomized variables. Psychometrika, 40(1), 5-32.

Coertjens, L., Van Daal, T., Donche, V., De Maeyer, S., Vanthounout, G., \& Van Petegem, P. (2013). Analysing change in learning strategies over time: A comparison of three statistical techniques. Evaluating Learning Pattern Development in Higher Education, 39(1), 49-55.

Corral de Zurita, N. \& Alcalá, M. T. (2002). Estrategias de aprendizaje y estudio de estudiantes universitarios. Corrientes:

Universidad Nacional del Nordeste. Recuperado de http://www.unne.edu.ar/unnev ieja/Web/cyt/cyt/2002/09-Educacion/D-03.pdf

Delgado Hurtado, M., \& Castrillo Lara, L. (2014). Efectividad del aprendizaje cooperativo en contabilidad: una contrastación empírica. Revista de Contabilidad. doi:10.1016/j.rcsar.2 014.05.003

Diseth, A. (2011). Self-efficacy, goal orientations and learning strategies as mediators between preceding and subsequent academic achievement. Learning and Individual Differences, 21(2), 191-195. doi: 10.1016/j.lin dif.2011.01.003

Doménech Betoret, F., Gómez Artiga, A., \& Lloret Segura, S. (2014). Personal variables, motivation and avoidance learning strategies in undergraduate students. Learning and Individual Differences, 35, 122-129. doi:10.1016/j.lindif.2014.06.007

Dunlosky, J., Rawson, K., Marsh, E., Nathan, M., \& Willingham, D. (2013). Improving students'learning with effective learning techniques: Promising directions from cognitive and educational psychology. Psychological Science Public Interest, 14(1), 4-58. doi: 10.1177/1529100612453266

Elosua Oliden, P. \& Zumbo, B. D. (2008). Coeficientes de fiabilidad para escalas de respuesta categórica ordenada. Psycothema, 20(4), 896-901.

Elvira Valdéz, M. A., \& Pujol, L. (2013). Autorregulación y rendimiento académico en estudiantes universitarios de reciente ingreso: Un estudio longitudinal. Educación y Futuro, 29, 205-219.

Fernández González, O. M., Martínez Conde Beluzán, M., \& Melipillán Araneda, R. (2009). Estrategias de aprendizaje y autoestima. Su relación con la permanencia y deserción universitaria. Estudios Pedagógicos, 35(1), 27-45.

Fernández Liporace, M., Scheinsohn, M.J., \& Uriel, F. (2010). Análisis factorial exploratorio del Inventario de Estrategias de aprendizaje y Estudio - LASSI. Memorias del I Congreso Internacional de Psicología y Educación $(C D)$. Panamá, Panamá: Psychology Investigation Corp. 
Flora, D. B., \& Curran, P. J. (2004). An empirical evaluation of altenative methods of estimation for confirmatory factor analysis with ordinal data. Psychol Methods, 9(4), 466-491.

Flowers, L. A., Bridges, B. K., \& Moore, J. L. (2011). Concurrent validity of the Learning and Study Strategies Inventory (LASSI): A study of African American precollege students. Journal of Black Studies, 20(10), 115. doi: 10.1177/0021934711410881

Gadermann, A. M., Guhn, M., \& Zumbo, B. D. (2012). Estimating ordinal reliability for Likert-type and ordinal item response data: A conceptual, empirical, and practical guide. Practical Assessment, Research \& Evaluation, 17(3), 1-13.

Gargallo, B., Almerich, G., Suárez Rodríguez, J.M., \& García Félix, E. (2012). Estrategias de aprendizaje en estudiantes universitarios excelentes y medios. Su evolución a lo largo del primer año de carrera. RELIEVE, 18(2). doi: 10.7203/relieve.18.2.2000

Goulão, M. F. (2014). The relationship of elearniner's with studies strategies to support learning. Procedia-Social and Behavioral Sciences, 116, 362-367.

Herrero, J. (2010). El análisis factorial confirmatorio en el estudio de la estructura y estabilidad de los instrumentos de evaluación: Un ejemplo con el Cuestionario de Autoestima CA-14. Psychosocial Intervention, 19(3), 289-300.

Holgado Tello, F., Chacón Moscoso, S., Barbero García, I., \& Vila Abad, E. (2010). Polychoric versus Pearson correlation in exploratory and confirmatory factor analysis of ordinal variables. Quality and Quantity, 44(1), 153166.

Jöreskog, K. G., \& Sörbom, D. (1999). LISREL 8: user's reference guide. Lincolnwood, IL: Scientific Software International, Inc.

Juras, J., \& Pasaric, Z. (2006). Aplication of tetrachoric and polychoric correlation coefficients to forecast verification. GEOFIZIKA, 23(1), 59-82.

Kálly, E. (2012). Learning strategiesand metacognitive awareness as predictors of academic achievment in a simple of Romanian second year students. Cognition,
Brain, Bahavior: An Interdisciplinary Journal, 16(3), 369-385.

Kesici, S., Baloglu, M., \& Deniz, M. (2011). Selfregulated learning strategies in relation with statistics anxiety. Learning and individual Differences, 21(4), 472-477. doi: http://dx.doi. org/10.1016/j.lindif.2011.02.006

Kesici, S., Sahin, I., \& Akturk, A. (2009). Analysis of cognitive learning strategies and computer attitudes according to college students' gender and locus of control. Computers in Human Behavior, 25, 529-534. doi:10.1016/j.chb.2008.11.004

Kisac, I., \& Budak, Y. (2014). Metacognitive strategies of the university students with respect to their perceived self-confidence levels about learning. Procedia-Social and Behavior Sciences, 116, 3336-3339. doi: 10.1016/j.sbspro.2014.01.759

Kline, R. B. (2005). Structural equation modeling. New York: Guilford Press.

Kovacova, L., \& Vackova, M. (2015). Implementation of e-learning into the process security education in universities. ProcediaSocial and Behavioral Sciences, 182, 414419. doi: : 10.1016/j.sbspro.2015.04.810

Lee, Y., \& Choi, J. (2013). A structural equation model of predictors of online learning retention. The Internet and Higher Education, 16, 36-42. doi: 10.1016/j.iheduc.2012.01.005

Lévy Mangin, J. P., \& González, N. (2006). Modelización y Causalidad. En: J. P. Lévy Mangin y J. Varela Mallou (Eds.), Modelización con estructuras de covacianzas en ciencias sociales (pp. 155-175). Coruña: Netbiblo.

López Martín, E., Expósito Casas, E., González, C., \& Jiménez García, E. (2012). Psychometric analysis of a scale on study skills and strategies: Evaluating and improving an adaptation of the LASSI inventory. Electronic Journal of Research in Educational Psychology, 10(3), 1383-1408.

Manzano Patiño, A., y Zamora Muñoz, S. (2009). Sistema de ecuaciones estructurales: Una herramienta de investigación. Cuaderno técnico 4. Centro Nacional de Evaluación para la Educación Superior A.C. Recuperado de http://www.senasica.gob.mx/includes/asp/dow nload.asp?iddocumento $=23068 \&$ idurl $=45367$ 
Martínez Arias, R. (2005). Psicometría: Teoría de los tests psicológicos y educativos. Madrid: Síntesis.

Merino Soto, C., \& Livia Segovia, J. (2009). Intervalos de confianza asimétricos para el índice la validez de contenido: Un programa Visual Basic para la $\mathrm{V}$ de Aiken. Anales de Psicología, 25(1), 169-171.

Moldasheva, G., \& Mahmood, M. (2014). Personality, learning strategies, and academic performance: Evidence from post-Soviet Kazakhstan. Education \& Training, 56(4), 343-359. doi: http://dx.doi.org/10.1108/ET10-2012-0101

Moreno Bayardo, M. G. (2000). Introducción a la Metodología de la Investigación Educativa 2. México, D.F.: Editorial Progreso.

Moreno Herrero, I. (2012). La web 2.0 como herramienta para la alfabetización digital en contextos multiculturales. Contextos, 27, 7993.

Namlu, A. G. (2004). Biliúötesi ö־renme stratejileri ölçme aracÕnÕn geliútirilmesi: Geçerlilik ve güvenilirlik çalÕúmasÕ. Anadolu Üniversitesi Sosyal Bilimler Dergisi, 4(2), 123-136.

Nieto Martín, S., \& Rodríguez Conde, J.M. (2010). Investigación y Evaluación Educativa en la Sociedad del Conocimiento. Salamanca: Ediciones Universidad de Salamanca.

Olejnik, S. \& Nist, S. L. (1992). Identifying latent variables measured by the Learning and Study Strategies Inventory (LASSI). Journal of Experimental Education, 62, 151-159.

Olivarez, A. \& Tallent, M. K. (1994). Psychometric properties of the Learning and Study Strategies Inventory-High School version. Journal of Experimental Education, 62, 243-257.

Peñalosa Castro, E., Landa Durán, P., \& Vega Valero, C.Z. (2006). Aprendizaje autorregulado: Una revisión conceptual. Revista Electrónica de Piscología Iztacala, 9(2), 1-21.

Pintrich, P. R., Smith, D. A., García, T., \& Mckeachie, W.J. (1991). A manual for the use of the Motivational Strategies for Learning Questionnaire (MSLQ). AnnArbor, MI: NCRIPTAL, the University of Michigan.
Prevatt, F., Petscher, Y., Proctor, B. E., Hurst, A., \& Adams, K. (2006). The Revised Learning and Study Strategies Inventory: An Evaluation of Competing Models. Educational and Psychological Measurement, 66(3), 448-458.

Rial Boubeta, A., Varela Mallou, J., Abalo Piñeiro, J., \& Lévy Mangin, J. P. (2006). El Análisis Factorial Confirmatorio. En: J.P. Lévy Mangin y J. Varela Mallou (Eds.), Modelización con estructuras de covarianzas en ciencias sociales (pp. 119-154). Coruña: Netbiblo.

Richaud de Minzi, M. C. (2005). Desarrollos del análisis factorial para el estudio de ítem dicotómicos y ordinales. Revista Interdisciplinaria, 22(2), 237-251.

Román, J. M., \& Gallego, S. (1994). Escala de Estrategias de Aprendizaje, ACRA. Madrid: TEA Ediciones.

Samuelstuen, M. S. (2003). Psychometric properties and item-key direction effects for the Learning and Study Strategies InventoryHigh School version with Norwegian students. Educational and Psychological Measurement, 63, 430-445.

Schermelleh-Engel, K., Moosbrugger, H., \& Müller, H. (2003). Evaluating the fit of structural equation models: Test of significance and descriptive goodness-of-fit measures. Methods of Psychological Research Online, 8(2), 23-74.

Sharifah, R. W. A., Khairiyah, M.Y., Haslenda, H., \& Zainura, Z. (2012). Sustainability education for first year engineering students using cooperative problem based learning. Procedia-Social and Behavioral Sciences, 56, 52-58.

Stevens, T., \& Tallent Runnels, M. K. (2004). The Learning and Study Strategies Inventory high school version: Issues of factorial invariance across gender and ethnicity. Educational and Psychological Measurement, 64(2), 332-346.

Stover, J. B., Uriel, F., \& Fernández Liporace, M. (2012). Inventario de estrategias de aprendizaje y estudio: Análisis psicométricos de una versión abreviada. Revista Argentina de Ciencias del Comportamiento, 4(3), 4-12. 
Sullivan, C., Eduljee, N., \& Pinkham, K. (2012). Motivational beliefs and use of learning strategies during the first year of college: $A$ longitudinal study. Proceedings of the 52nd Annual Meeting of the New England Psychological Association. doi: http://dx.doi.org/10.1037/e541672013-046

Tirziu, A. M., \& Vrabie, C. (2015). Education 2.0: E-learning methods. Procedia-Social and Behavioral Sciences, 186, 376-380. doi: 10.1016/j.sbspro.2015.04.213

Uriel, F., Stover, J. B., Scheinsohn, M. J., Contini de González, N., \& Fernández Liporace, M.M. (2009). Escala para la evaluación de estrategias de estudio y aprendizaje (Adaptación UBA-CONICET). Buenos Aires: Departamento de Publicaciones, Facultad de Psicología, UBA.

Valderrey Sanz, P. (2010). SPSS 17. Extracción del conocimiento a partir del análisis de datos. Madrid: RA-MA.

Valentín, A., Mateos, P. M., González Tablas, M. M., Pérez, L., López, E., \& García, I. (2013). Motivation and learning strategies in the use of ICTs among university students. Computers \& Education, 61, 52-58. doi: 10.1016/j.compedu.2012.09.008

Valle, A., \& Rodríguez, S. (1998). Estrategias de aprendizaje y rendimiento académico. Boletín de Psicología, 60, 27-53.

Vallejo Seco, G. (1992). Análisis Multivariantes Aplicados a las Ciencias del Comportamiento. Oviedo: Universidad de Oviedo.

Van Seters, J. R., Ossevoort, M. A., Tramper, J. \& Goedhart, M.J. (2012). The influence of students' characteristics on the use of adaptive e-learning material. Computers \& Education, 58(3), 942-952. doi:10.1016/j.compedu. 2011. 11.002

Weinstein, C. E., \& Mayer, R. E. (1986). The teaching of learning strategies. In M.C. Wittrock (Ed.), Handbook of research on teaching (pp. 315-327). New York: McMillan.

Weinstein, C. E., \& Meyer, D. K. (1991). Cognitive learning strategies and college teaching. New Directions for Teaching and Learning, 45, 15-26. doi: 10.1002/t1.37219914 505

Weinstein, C. E., \& Palmer, D. R. (2002). Learning and Study Strategies Inventory

Revista Iberoamericana de Diagnóstico y Evaluación - e Avaliação Psicológica. RIDEP · N44 · Vol.2 · 116-130 2017
(LASSI): User's manual (2nd ed.). Clearwater, FL: H \& H Publishing.

Weinstein, C. E., Ridley, D. S., Dahl, T., \& Weber, E.S. (1989). Helping students develop strategies for effective learning. Educational Leardership, 46(4), 18-19.

Weinstein, C. E. Schulte, A. C., \& Palmer, D. R. (1987). Learning and Study Strategies Inventory (LASSI). Clearwater. $\mathrm{F} 1: \mathrm{H} \& \mathrm{H}$ Publishing.

Wenbin, G. (2006). A study of the self-regulated learning strategies and levels of achievement of female junior college students. Psychological Science, 29(3), 721-723.

Wu Yuin, H., Rustam, S., Chin Yu, W., \& Zhi Huan, H. (2012). A pilot study of cooperative programming learning behavior and its relationship with students 'learning performance. Computers and Education, 58(4), 1267-1281.

Yip, M. (2009). Differences between high and low academic achieving university students in learning and study strategies: A further investigation. Educational Research and Evaluation, 15(6), 561-570. doi: http://dx.doi.org/10.1080/1380361090335471 8

Yip, M. C. W. (2013). The reliability and validity of the chinese version of the Learning and Study Strategies Inventory (LASSI-C). Journal of Psychoeducational Assessment, 31(4), 396-403.

Zimmerman, B., \& Martínez Pons, M. (1988). Construct validation of a strategy model of student self-regulated learning. Journal of Educational Psychology, 80(3), 22-63. 